\title{
Interaction between Perirhinal and Medial Prefrontal Cortex Is Required for Temporal Order But Not Recognition Memory for Objects in Rats
}

\author{
Darren K. Hannesson, ${ }^{1}$ John G. Howland, ${ }^{2}$ and Anthony G. Phillips ${ }^{2}$ \\ ${ }^{1}$ Department of Psychology, University of Regina, Regina, Saskatchewan, Canada S4S 0A2, and ${ }^{2}$ Departments of Psychology and Psychiatry, University of \\ British Columbia, Vancouver, British Columbia, Canada V6T 2A1
}

\begin{abstract}
The present study investigated the roles of the perirhinal cortex, medial prefrontal cortex, and intrahemispheric interactions between them in recognition and temporal order memory for objects. Experiment 1 assessed the effects of bilateral microinfusions of the sodium channel blocker lidocaine into either the anterior perirhinal or medial prefrontal cortex immediately before memory testing in a familiarity discrimination task and a recency discrimination task, both of which involved spontaneous exploration of objects. Inactivation of the perirhinal cortex disrupted performance in both tasks, whereas inactivation of the medial prefrontal cortex disrupted performance in the recency, but not the familiarity, discrimination task. In a second experiment, the importance of intrahemispheric interactions between these structures in temporal order memory were assessed by comparing the effects of unilateral inactivation of either structure alone with those of crossed unilateral inactivation of both structures on the recency discrimination task. Crossed unilateral inactivation of both structures produced a significant impairment, whereas inactivation of either structure alone produced little or no impairment. Collectively, these findings suggest that the perirhinal cortex, but not the medial prefrontal cortex, contributes to retrieval of information necessary for long-term object recognition, whereas both structures, via intrahemispheric interactions between them, contribute to retrieval of information necessary for long-term object temporal order memory. These data are consistent with models in which attributed information is stored in posterior cortical sites and supports lower-order mnemonic functions (e.g., recognition memory) but can also be retrieved and further processed via interactions with the prefrontal cortex to support higher-order mnemonic functions (e.g., temporal order memory).
\end{abstract}

Key words: anterior cingulate; prelimbic cortex; memory systems; retrieval; object memory; temporal order memory

\section{Introduction}

The ability to discriminate familiar from novel stimuli is the most basic activity supported by recognition memory and is widely used as an assay for this form of memory. When stimuli are complex objects, familiarity discrimination relies critically on the perirhinal cortex (Prh). Thus, in both primates and rats, lesions of Prh consistently disrupt performance on familiarity discrimination tasks with objects (Aggleton and Brown, 1999; Murray and Richmond, 2001), and recordings from Prh neurons reveal experience-modifiable object-selective unit activity (Brown and Bashir, 2002). However, other evidence suggests that the prefrontal cortex (PFC) may also play an important role in familiarity discriminations with objects. For example, in both primates and rats, lesions of the PFC disrupt performance on some object fa-

Received Dec. 15, 2003; revised Feb. 25, 2004; accepted March 29, 2004.

This work was supported by a Natural Sciences and Engineering Research Council (Canada) (NSERC) operating grant awarded to A.G.P., an NSERC postdoctoral fellowship awarded to D.K.H., and a K. M. Hunter/Canadian Institutes of Health Research doctoral training fellowship awarded to J.G.H. Our gratitude is extended to Deana Chavez, Erin MacKenzie, and Tonia Yim for assistance with various components of these studies.

Correspondence should be addressed to Dr. Anthony G. Phillips, Department of Psychiatry, University of British Columbia, 2255 Westbrook Mall, Vancouver, British Columbia, Canada V6T 2A1. E-mail: aphillips@psych.ubc.ca. D01:10.1523/JNEUROSCI.5517-03.2004

Copyright $\odot 2004$ Society for Neuroscience $\quad$ 0270-6474/04/244596-09\$15.00/0 miliarity discrimination tasks (Meunier et al., 1997; Ragozzino et al., 2002), whereas recordings from PFC neurons also reveal experience-modifiable object-selective unit activity (Rainer and Miller, 2000).

Temporal order memory, defined as memory for the order in which items/events have been experienced, represents another important form of memory, which in its simplest application supports the ability to discriminate the relative recency of stimuli/events. Recency discrimination, across a range of stimulus materials, relies critically on the PFC, as indicated by studies with humans, monkeys, and rats in which damage to the PFC impairs recency discrimination performance in tasks using verbal, spatial, olfactory, or visual stimuli (Shimamura et al., 1990; Petrides, 1991; Chiba et al., 1994; Kopelman et al., 1997; Mitchell and Laiacona, 1998; Kesner et al., 2002). Furthermore, recordings from PFC neurons in monkeys reveal experience-modifiable order-selective unit activity (Funahashi et al., 1997; Ninokura et al., 2003). In contrast, the role of the Prh in temporal order memory is unknown, but presumably to the extent that recency judgments rely on object recognition-related functions, the Prh should also be involved. In this regard, it is noteworthy that a subset of Prh neurons exhibits a response decrement to recently, but not more remotely, experienced objects and therefore could 
contribute to recency discrimination in this manner (Brown, 1996).

The present study was undertaken to investigate the relative roles of the Prh and medial PFC (mPFC) in the rat in object recognition and temporal order memory. Behavioral testing used two tests of spontaneous object exploration, one requiring familiarity discrimination (Ennaceur and Delacour, 1988) and the other recency discrimination (Mitchell and Laiacona, 1998), which were chosen because they do not require extensive training, rule learning, or the use of primary reinforcement and, thus, avoid potential confounds related to effects on these aspects of performance. In experiment 1 , the anterior Prh or mPFC was inactivated bilaterally using local microinfusions of the sodium channel blocker lidocaine immediately before the memory testing trial in both tasks. This enabled selective assessment of contributions to retrieval of object familiarity or recency information. The results from experiment 1 demonstrated that the Prh was involved in object recognition memory, whereas both the Prh and mPFC were involved in object temporal order memory. Thus, in experiment 2, the effects of crossed unilateral inactivation of the anterior Prh and mPFC immediately before memory testing in the recency discrimination task were assessed to determine the importance of intrahemispheric interactions between these structures in the retrieval of object temporal order memory. We have previously successfully used similar crossed inactivations in investigating neural circuits mediating spatial memory (Floresco et al., 1997, 1999).

\section{Materials and Methods Experiment 1 \\ Subjects}

Thirty-three Long-Evans hooded rats (Charles River Laboratories, Montreal, Quebec, Canada), weighing 325-375 gm at the beginning of the study, were used as subjects. Food and water were available ad libitum throughout the experiment. Rats were maintained in pairs in Plexiglas cages throughout the experiment. All experimental procedures were performed during the light portion of the $12 \mathrm{hr}$ light/dark cycle. All rats were handled $5 \mathrm{~d}$ per week throughout the experiment.

Rats were divided into two independently tested groups that received cannulas implanted bilaterally into either the anterior $\operatorname{Prh}(n=13)$ or the $\operatorname{mPFC}(n=20)$. Note that larger numbers of rats were used in the mPFC group to ensure sufficient power was available to observe any effect on the familiarity discrimination task (see Results). For both of these groups of rats, a within-subjects design was used in which each rat received two tests in each of two tasks (see below), one after receiving a sham infusion and one after receiving a lidocaine infusion. Thus, a total of four cells were used with task (familiarity discrimination or recency discrimination) and infusion (sham or lidocaine) as independent variables.

\section{Surgery}

In preparation for surgery, rats were anesthetized with $100 \mathrm{mg} / \mathrm{kg}$ ketamine hydrochloride and $7 \mathrm{mg} / \mathrm{kg}$ xylazine. The rat's head was shaved and placed in a stereotaxic apparatus before having the skin retracted and skull leveled. Twenty-three gauge stainless steel guide cannulas were implanted bilaterally at the following coordinates relative to skull at bregma: anterior Prh: $-4.0 \mathrm{~mm}$ [anterior-posterior (AP)], $\pm 5.0 \mathrm{~mm}$ [mediolateral $(\mathrm{ML})$ ], $-7.4 \mathrm{~mm}$ [dorsoventral (DV)], manipulator arm angled $10^{\circ}$ away from midline; mPFC: $+2.9 \mathrm{~mm}$ (AP), $\pm 0.7 \mathrm{~mm}$ (ML), $-4.0 \mathrm{~mm}(\mathrm{DV})$. The cannula tips were targeted at the border between the temporal association cortex and the dorsal Prh or at the dorsalmost region of the prelimbic cortex in the two groups, respectively. Four jeweler's screws and dental acrylic were used to secure the cannulas in place. Thirty gauge obdurators flush with the tips of the cannulas were inserted to prevent entry of materials into the brain. Each rat was housed individually for $4 \mathrm{~d}$ after surgery and then re-housed in pairs for at least $3 \mathrm{~d}$ before the start of habituation training.

\section{Behavioral testing}

Testing environment, apparatus, and objects. Behavioral testing took place in a rectangular windowless room with one door. The walls were painted an off-white color and were hung with numerous posters. Overhead ventilation fans produced background noise. For data acquisition, an overhead video camera connected to a video cassette recorder was used to record movement of rats in the open field.

A square open-field box $[60 \times 60 \times 45$ (height) $\mathrm{cm}]$ constructed from white corrugated plastic was used for all testing. At one end of the box, two $10 \mathrm{~cm}$ square "object mounts" constructed from white Lego were glued in position $3 \mathrm{~cm}$ from the back wall and $12 \mathrm{~cm}$ from the side walls.

The stimuli used in testing in the open field were objects constructed from Lego. For habituation testing, $10 \times 10 \times 4$ (height) $\mathrm{cm}$ rectangular white objects were used. For memory testing, 12 unique objects that ranged in size from $10 \times 10 \times 8$ (height) $\mathrm{cm}$ to $12 \times 12 \times 10$ (height) $\mathrm{cm}$ were used. These were constructed from combinations of pieces of three different colors (blue, red, yellow, or green) and were made in triplicate. The shape and pattern of each object was distinct and selected on the bases of pilot study data indicating that normal rats could discriminate between them.

Behavioral paradigms. Object memory testing was achieved using two previously reported tasks relying on spontaneous exploration of objects in an open field (Ennaceur and Delacour, 1988; Ennaceur and Aggleton, 1997; Mitchell and Laiacona, 1998). In the familiarity discrimination version of the task, rats received a training trial in which they were placed in the open field with two copies of a novel object and allowed to explore for $4 \mathrm{~min}$. After a delay of $105 \mathrm{~min}$, rats received a test trial identical to the training trial, except that a third copy of the original object (the "familiar" object) and a second novel object were used. On this test trial, normal rats spend more time exploring the novel object than the familiar object, a feat that requires the rat to recognize the previously explored object, and, hence, this task is widely used to assess object recognition memory (Ennaceur and Aggleton, 1997). In the recency discrimination version of the task, rats received a training trial in which they were placed in an open field with two copies of a novel object and allowed to explore for $4 \mathrm{~min}$ (Mitchell and Laiacona, 1998). After a delay of $60 \mathrm{~min}$, rats received a second training trial identical to the first, except that two copies of a new novel object were present. After an additional delay of $45 \mathrm{~min}$, rats received a test trial identical to the training trials, except that one copy of the object from trial 1 (the "old familiar" object) and one copy of the object from trial 2 (the "recent familiar" object) were present. On this test trial, normal rats spend more time exploring the old familiar object than the recent familiar object, a feat that requires the rat to both recognize the previously explored objects and to discriminate their relative recency, and, hence, this task is used to assess object temporal order memory (Mitchell and Laiacona, 1998).

One possible explanation for the expected performance in the recency discrimination task is that the old familiar object is forgotten and thus functions like a novel object. This would reduce the demands of the task to simply performing a familiarity discrimination. Furthermore, in the recency discrimination task, retroactive interference attributable to experience with the recent familiar object could make retrieval of memory for the old familiar object more difficult. To assess these possibilities, we ran a pilot study using a three-trial familiarity discrimination task that was identical to the recency discrimination task, except that in the memory test trial, one object was a third copy of the object from trial 1 (the old familiar object) and the other object was novel. We hypothesized that if memory for the old familiar object was intact, rats would spend more time exploring the novel than the old familiar object. Our data confirmed that normal unoperated rats showed a preference for a novel object over an old familiar object $(n=10, \mathrm{D} 1=4.9 \mathrm{sec}, p<0.05$; D2 $=0.171, p<$ 0.05 ; see below for an explanation of D1 and D2 measures), thus demonstrating intact memory for the old familiar object. This finding indicates that the recency discrimination task does indeed require an assessment of the relative recency of the two remembered objects and not simply an assessment of relative familiarity between a recently explored object and a novel (i.e., forgotten) object.

In the present transient lesion experiment, memory testing then used the two-trial familiarity discrimination task and the recency discrimina- 
tion task. The standard familiarity discrimination task was chosen over the three-trial familiarity discrimination task described above because it was deemed to be a purer test of recognition memory, devoid of potential interpretational confounds related to interference effects in the threetrial version. This is of particular concern because temporal order memory could assist with minimizing interference effects on recognition memory by assisting in differentiating successive episodes of experience with different objects. Thus, changes in temporal order memory might be associated with concomitant effects on the three-trial familiarity discrimination task that could confound clear interpretation of changes in performance across the two tasks (Kesner and Holbrook, 1987; Lalonde and Hannequin, 1999).

\section{Procedures and testing schedule}

Before any memory testing, rats received four habituation sessions, each given on separate days within an $8 \mathrm{~d}$ period. In preparation for each session, a squad of between six and eight rats was brought from the colony room to a holding room. Both the open field and the objects (white, $10 \times 10 \times 4 \mathrm{~cm}$ ) were then prepared as follows. They were sprayed with a solution of $50 \%$ alcohol, wiped thoroughly with a sponge soaked in distilled water, and then dried with paper towels. The objects were then attached to the mounts. Rats were then brought to the testing room individually and placed in the open field facing the center of the wall opposite the objects. Five minutes later, the experimenter returned, removed the rat from the maze, and returned it to the holding room. Each additional rat in the squad received an identical habituation session, with the maze and objects thoroughly cleaned between sessions, until all rats had been tested. The squad was then returned to the colony room.

Memory testing began between 48 and $96 \mathrm{hr}$ after habituation was completed. Rats received two familiarity discrimination and two recency discrimination tests each, once after a sham infusion and once after a lidocaine infusion, and were tested with at least 1 week between each session. The order was quasi counter-balanced. In general, the testing procedures for memory trials in both the familiarity and recency discrimination tasks were similar to habituation trials. Before each testing session, a squad of rats, the objects, and the open field were washed and dried. Trials were administered as in habituation trials, except that they were only $4 \mathrm{~min}$ in duration. Between trials, rats remained in the holding room until all trials of that testing session were completed for the squad. Objects for each trial were selected pseudo-randomly according to the following criterion: no rat was ever tested with the same object more than once (except as a familiar object on a test trial), several object pairings were used within each treatment cell, and these pairings were matched across treatment cells.

\section{Microinfusion procedure}

On the same days as habituation to the open field, rats were habituated to the infusion procedure. In the first habituation session, rats were brought individually to the infusion room, had their obdurators removed, and were placed in the infusion box $[20 \times 20 \times 30$ (height) $\mathrm{cm}$ made from clear Plexiglas] for $4 \mathrm{~min}$. On the second through fourth days, rats also had 30 guage stainless steel sham injection needles inserted bilaterally until they were flush with the ends of the implanted guide cannulas before being placed in the infusion box for $4 \mathrm{~min}$.

On memory testing days, rats were brought to the infusion room between 10 and $15 \mathrm{~min}$ before the final trial of testing in either the familiarity or recency discrimination task. Obdurators were removed, 30 gauge stainless steel injection needles were inserted bilaterally, and rats were placed in the infusion box. For lidocaine trials, needles were used that extended $0.8 \mathrm{~mm}$ beyond the tips of the guide cannulas, and $4 \%$ lidocaine (32 $\mu \mathrm{g}$ in $0.8 \mu \mathrm{l}$ of $0.9 \%$ saline) was delivered at a rate of 0.4 $\mu \mathrm{l} / \mathrm{min}$ for $2 \mathrm{~min}$ via a Hamilton microsyringe driven using a Harvard pump. Injection needles were left in place for $1 \mathrm{~min}$ after the infusion to allow for diffusion, after which the rat was removed from the box and returned to the holding room. These infusion parameters were chosen to produce significant inactivation of either the anterior Prh or the mPFC. For the Prh infusions, both areas 35 and 36 of the Prh were targeted for inactivation (for definition of Prh borders, see Burwell, 2001). Anterior rather than posterior inactivations were selected because projections to the $\mathrm{mPFC}$ are stronger from this region of the Prh (Delatour and Witter, 2002; McIntyre et al., 1996), which was important because we were interested in exploring potential Prh-mPFC interactions in experiment 2. For the mPFC infusions, the prelimbic cortex was the central target, but substantial inactivation in the anterior cingulate cortex was also anticipated. The size and subregional targets of the mPFC infusions were chosen because previous research had implicated these regions in temporal order memory in the rat (Chiba et al., 1994; Mitchell and Laiacona, 1998) and had also shown that effects on temporal order memory were positively related with lesion size within the mPFC (Chiba et al., 1994). Sham trials were identical to lidocaine trials, except that shorter needles that were flush with the end of the guide cannulas were used, and no infusion was made (although the syringe pump was run for $2 \mathrm{~min}$ as in lidocaine trials).

\section{Histology}

After behavioral testing, rats were killed with an overdose of sodium pentobarbital and perfused transcardially with $9 \%$ saline. Brains were fixed in formalin and then frozen before $50 \mu \mathrm{m}$ coronal sections were taken throughout the extent of the Prh or PFC. Every second section in the region of the cannulas was mounted and stained with cresyl violet. The location of the cannulas was documented by matching sections with relevant plates from Paxinos and Watson (1998). For Prh placements, definitions of borders with other structures were based on data from Burwell (2000).

\section{Data scoring and analysis}

Time spent exploring each of the two objects available on a given trial was scored from videotape with stopwatches. A rat was judged to be actively exploring an object when its nose was directed within $2 \mathrm{~cm}$ at an object and either its head or vibrissa was moving. Three dependent measures were used based on previous studies of spontaneous object exploration (Ennaceur and Delacour, 1988). The first was exploration times of the objects on a given trial. These data were then used to calculate two additional measures that were taken as indices of object preferences on test trials. The first was the difference score (D1) calculated as the difference in time spent exploring the novel versus the familiar object (familiarity discrimination task) or the old familiar versus the recent familiar object (recency discrimination task). The second was the weighted difference score (D2) calculated as D1/time spent exploring both objects.

Because the groups were run independently and the comparisons we were interested in were within groups (e.g., the effects of lidocaine vs sham infusions in the Prh) rather than between groups (e.g., the effects of sham Prh infusions vs sham mPFC infusions), data from the Prh and mPFC groups were analyzed separately. Overall, exploration times across trials in each task were analyzed first using repeated-measures ANOVA with trial and infusion (sham vs lidocaine) as factors. Follow-up analyses were performed with two-tailed within-groups $t$ tests. For test trial data, D1 and D2 measures were subjected to a two-way repeated-measures ANOVA with task (familiarity discrimination vs recency discrimination) and infusion (sham vs lidocaine) as factors. Additional comparisons were made using one-tailed single group $t$ tests (with a comparison value of 0 indicating equal exploration of the two objects as predicted by chance performance) and within-groups $t$ tests. One-tailed tests were used because we hypothesized a priori that rats would spend more time exploring the novel object in the familiarity discrimination task and the old familiar object in the recency discrimination task and that lidocaine-infused rats would perform more poorly relative to sham-infused rats.

\section{Experiment 2}

\section{Subjects and surgery}

Eleven Long-Evans hooded rats (Charles River Laboratories) were used as subjects and held under the same conditions as in experiment 1 .

Rats were implanted with cannulas unilaterally in the anterior Prh and mPFC in opposite hemispheres using surgical procedures as in experiment 1 . A within-subjects design was used in which each rat received four tests in the recency discrimination task and received a sham infusion, unilateral mPFC lidocaine infusion, unilateral Prh lidocaine infusion, or a combined unilateral Prh and mPFC lidocaine infusion in counter- 
balanced order. Thus, a total of four cells were used with infusion (sham, mPFC, Prh, or mPFC plus Prh) as the independent variable.

\section{Behavioral testing}

Habituation and behavioral testing were the same as in experiment 1 , with the exception that the recency discrimination task was used in all four tests.

\section{Microinfusion procedure and histology}

General infusion procedures including habituation were the same as in experiment 1 . Specific to experiment 2 , each rat received four different testing sessions that included a sham, mPFC lidocaine, Prh lidocaine, or mPFC plus Prh lidocaine infusion administered 10-15 min before the testing trial (i.e., the third trial) in the recency discrimination task. In sham trials, sham needles were inserted into both the mPFC and Prh cannulas. In mPFC and Prh trials, a lidocaine infusion was made into one structure and a sham infusion at the other. In mPFC plus Prh trials, a lidocaine infusion was made into both sites.

On completion of the study, the location of the cannulas in each rat was assessed using histological methods as in experiment 1.

\section{Data scoring and analysis}

Data were scored as in experiment 1 . Analysis of the total amount of object exploration across trials used repeated-measures ANOVA with trial (three levels) and infusion (four levels) as independent variables. Follow-up analyses were performed with two-tailed within-groups $t$ tests. For test trial data, D1 and D2 measures were subjected to repeated measures ANOVA with infusion (sham, mPFC, Prh, mPFC plus Prh) as the independent variable. Additional comparisons were made using onetailed single-group $t$ tests (with a comparison value of 0 indicating equal exploration of the two objects as predicted by chance performance) and within-groups $t$ tests. One-tailed tests were used because we hypothesized $a$ priori that rats would spend more time exploring the old familiar arm in the recency discrimination task and that the infused groups would perform more poorly than the sham group and that the mPFC plus Prh group would perform more poorly than the unilaterally infused group.

\section{Results}

\section{Experiment 1}

Histology

All rats included in behavioral analyses in the mPFC group $(n=$ 20) had bilateral guide cannulas with needle tips found in the prelimbic region of the medial PFC between 2.2 and $3.2 \mathrm{~mm}$ anterior to bregma (Fig. 1). Based on previous estimates of the functional spread of lidocaine using similar volumes (Seamans et al., 1995) and on research indicating the fully effective spread of lidocaine generally conforms to the relation $r=(3 \times V / 4 \times \pi)^{1 / 3}$, where $r$ equals the effective radius and $V$ equals the injected volume (Tehovnik and Sommer, 1997), these infusions were likely to have substantially reduced neural activity in a radius between 0.8 and $1.3 \mathrm{~mm}$ from the infusion site. This would have lead to inactivation of substantial portions of the prelimbic cortex, smaller portions of the anterior cingulate cortex, and dorsal portions of infralimbic cortex in most rats. In terms of time course, inactivations would have reached peak onset within 3 or 4 min and returned to near baseline within 25-30 min after the start of infusions (Tehovnik and Sommer, 1997), thus ensuring that all behavioral testing, which took place between 5 and $15 \mathrm{~min}$ after infusions, occurred during the period of maximal inactivation.

All rats included in behavioral analyses in the Prh group $(n=$ 13) had bilateral guide cannulas with needle tips found between 3.6 and $4.16 \mathrm{~mm}$ posterior to bregma in the border region between the temporal association cortex and area 36 of the Prh within cortical layers 3-6 (Fig. 2). Based on considerations outlined above about the spread of lidocaine, we estimate that the infusions substantially reduced activity in the full dorsal-ventral extent of the anterior Prh (typically from $\sim 2.8$ to $4.8 \mathrm{~mm}$ poste-

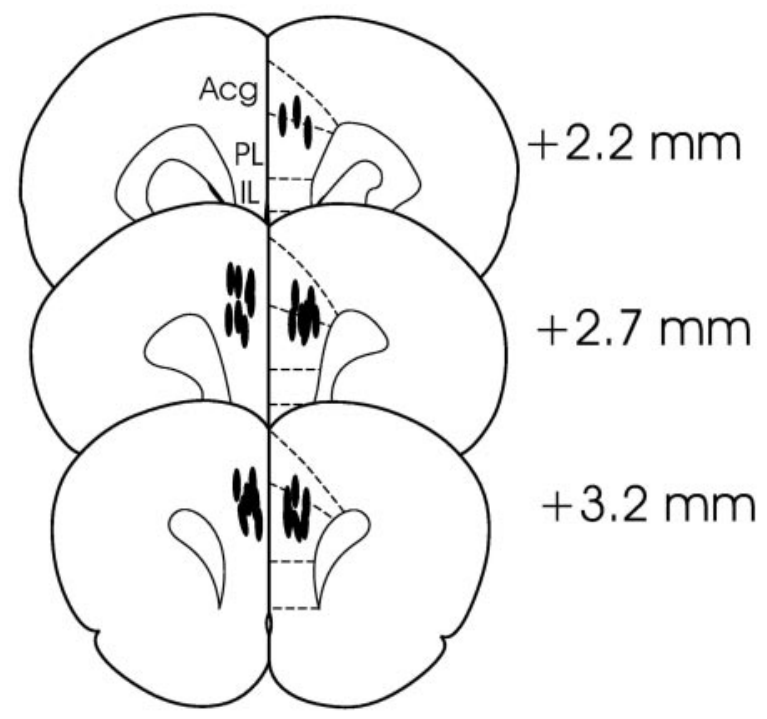

Figure 1. Location of the injection needles (lines) in rats in the mPFC group included in the behavioral analyses (plates located anterior to bregma and adapted from Paxinos and Watson, 1998). Acg, Anterior cingulate cortex; IL, infralimbic cortex; PL, prelimbic cortex.

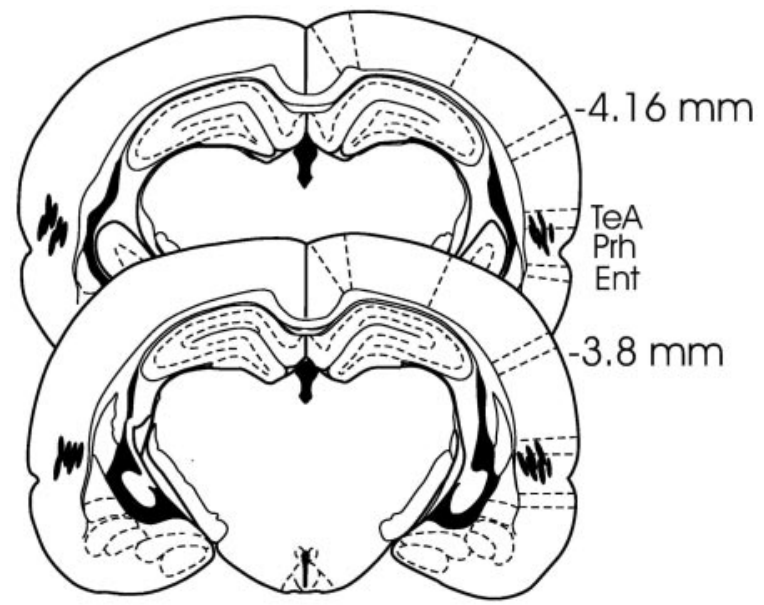

Figure 2. Location of the injection needles (lines) in rats in the Prh group included in the behavioral analyses (plates located posterior to bregma and adapted from Paxinos and Watson, 1998). Ent, Entorhinal cortex; Prh, perirhinal cortex; TeA, temporal association cortex.

rior to bregma) and portions of the anterior lateral entorhinal cortex and/or temporal association cortex.

\section{Behavioral testing}

Raw exploration times across trials for both the MPFC and Prh groups in both tasks are presented in Table 1. In general, most rats spent between 20 and $45 \mathrm{sec}$ in active exploration of the objects in both tasks, and this tended to be consistent across trials within tasks as indicated by the absence of a trial effect (familiarity discrimination task: mPFC group: $F_{(1,34)}=0.403, p=0.530$; Prh group: $F_{(1,24)}=2.229, p=0.148$; recency discrimination task: mPFC group: $F_{(2,68)}=2.375, p=0.101$; Prh group: $F_{(2,48)}=$ 2.097, $p=0.134$ ) or a trial by infusion interaction (familiarity discrimination task: $\mathrm{mPFC}$ group: $F_{(1,34)}=0.070, p=0.792 ; \mathrm{Prh}$ group: $F_{(1,24)}=0.862, p=0.362$; recency discrimination task: mPFC group: $F_{(2,68)}=1.385, p=0.257$; Prh group: $F_{(2,48)}=$ $1.072, p=0.350$ ). Overall, the above data suggest that infusions did not produce a gross impairment in exploratory behavior. 
Table 1. Exploration times (in seconds; mean \pm SEM) by the Prh- and mPFC-implanted rats after sham or lidocaine infusions in experiment 1 across trials in the familiarity and recency discrimination tasks

\begin{tabular}{|c|c|c|c|c|c|}
\hline Task plus infusion & Trial 1 total & Trial 2 total & Trial 3 total & Trial 3 single object & Trial 3 single object \\
\hline RcgM plus sham Prh & $31.6 \pm 3.4$ & $\mathrm{~N} / \mathrm{A}$ & $30.2 \pm 2.9$ & $18.8 \pm 2.4($ object N) & $11.4 \pm 1.1$ (object F) \\
\hline RcgM plus Lido Prh & $35.8 \pm 3.8$ & $\mathrm{~N} / \mathrm{A}$ & $29.7 \pm 3.3$ & $15.2 \pm 1.9$ (object N) & $14.6 \pm 6.0$ (object F) \\
\hline RcgM plus sham mPFC & $33.6 \pm 3.1$ & $\mathrm{~N} / \mathrm{A}$ & $30.8 \pm 2.6$ & $18.0 \pm 1.7$ (object N) & $12.6 \pm 0.9$ (object F) \\
\hline RcgM plus Lido mPFC & $35.6 \pm 3.2$ & $\mathrm{~N} / \mathrm{A}$ & $34.5 \pm 4.4$ & $19.4 \pm 2.3$ (object N) & $14.8 \pm 2.0$ (object F) \\
\hline TM plus sham Prh & $33.6 \pm 3.6$ & $31.7 \pm 5.3$ & $33.8 \pm 4.9$ & $20.0 \pm 3.1\left(\right.$ object $\left.F_{0}\right)$ & $13.6 \pm 2.0\left(\right.$ object $\left.F_{R}\right)$ \\
\hline TM plus Lido Prh & $33.4 \pm 3.2$ & $30.0 \pm 4.6$ & $40.2 \pm 5.2$ & $19.0 \pm 2.2\left(\right.$ object $\left.F_{0}\right)$ & $21.0 \pm 3.2\left(\right.$ object $\left.F_{R}\right)$ \\
\hline TM plus sham mPFC & $30.3 \pm 2.2$ & $25.4 \pm 2.4$ & $31.6 \pm 3.4$ & $17.7 \pm 1.6\left(\right.$ object $\left.F_{0}\right)$ & $13.6 \pm 1.6\left(\right.$ object $\left.F_{R}\right)$ \\
\hline TM plus Lido mPFC & $28.6 \pm 2.3$ & $24.2 \pm 1.9$ & $24.5 \pm 2.7$ & $12.2 \pm 1.5\left(\right.$ object $\left.F_{0}\right)$ & $12.9 \pm 1.7$ (object $\left.F_{R}\right)$ \\
\hline
\end{tabular}

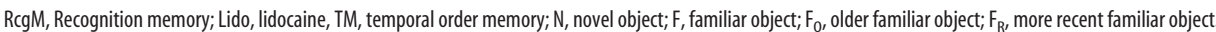
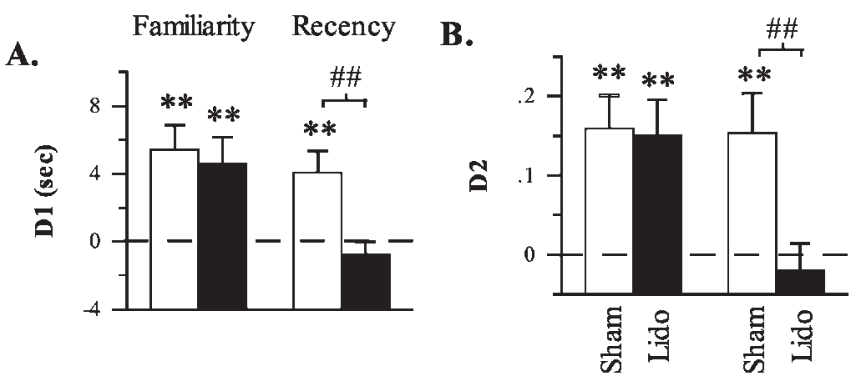

Figure 3. Differences in the amount of exploration directed at novel versus familiar (familiarity discrimination task) or old familiar versus recent familiar (recency discrimination task) objects after sham or lidocaine (Lido) infusions into the mPFC in experiment 1. $A$, Difference score (D1) calculated as time spent exploring the novel (or old familiar) object less time spent exploring the familiar (or recent familiar) object. $B$, Weighted difference score (D2) calculated as D1 divided by the time spent exploring both objects. ${ }^{* *} p<0.01$, one-tailed, relative to chance performance (i.e., 0 , indicated by the dashed line); \#\# $<0.01$, one-tailed, sham versus lidocaine.
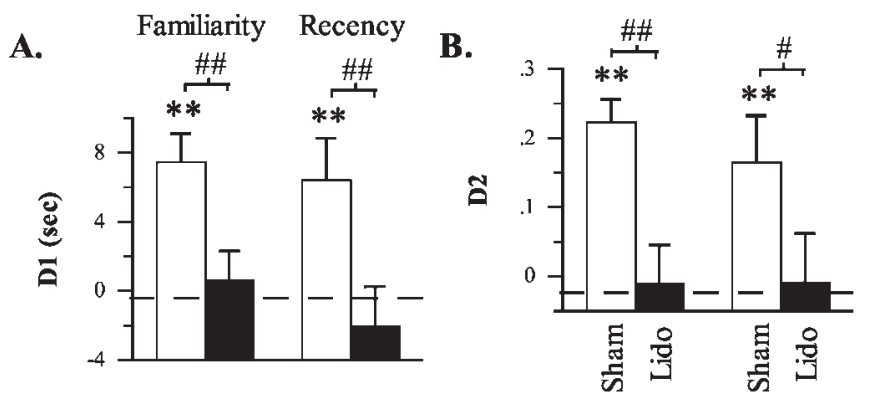

Figure 4. Differences in the amount of exploration directed at novel versus familiar (familiarity discrimination task) or old familiar versus recent familiar (recency discrimination task) objects after sham or lidocaine (Lido) infusions into the anterior Prh in experiment 1. A, Difference score (D1) calculated as time spent exploring the novel (or old familiar) object less time spent exploring the familiar (or recent familiar) object. $B$, Weighted difference score (D2) calculated as D1 divided by the time spent exploring both objects. ${ }^{* *} p<0.01$, one-tailed, relative to chance performance (i.e., 0 , indicated by the dashed line); $\# p<0.05$ and \#\#p $<0.01$, one-tailed, sham versus lidocaine.

On test trials, data indicated that lidocaine infusions into the mPFC selectively disrupted the object recency discrimination task, whereas infusions into the Prh disrupted both the familiarity and recency discrimination tasks (Table 1; Figs. $3 A, B, 4 A, B$ ). The disruptive effect of lidocaine infusions into the $\mathrm{mPFC}$ was indicated by a significant effect of infusion in terms of both D1 $\left(F_{(1,19)}=4.463 ; p=0.048\right)$ and D2 $\left(F_{(1,19)}=5.385 ; p=0.032\right)$ measures. Initial analyses also provided some indication that this effect differed between the recency and familiarity discrimination tasks with a near significant task by infusion interaction in terms of the D2 measure $\left(F_{(1,19)}=3.911 ; p=0.063\right)$ but not the D1 measure $\left(F_{(1,19)}=1.697 ; p=0.208\right)$. Subsequent planned comparisons, however, strongly indicated that the recency and familiarity discrimination tasks were differentially affected by $\mathrm{mPFC}$ infusions as shown by a significant infusion effect in the recency, but not the familiarity, discrimination task in terms of both D1 (recency discrimination task: $t_{(19)}=3.33, p=0.002$; familiarity discrimination task: $t_{(19)}=0.320, p=0.376$ ) and D2 (recency discrimination task: $t_{(19)}=3.282, p=0.002$; familiarity discrimination task: $\left.t_{(19)}=0.130, p=0.449\right)$ measures. Consistent with these findings, in the recency discrimination task, rats exhibited a significantly above chance bias for the old familiar object after sham, but not lidocaine, infusions (D1: sham: $t_{(19)}=3.357, p<$ 0.002; lidocaine: $t_{(19)}=-0.997, p>0.170$; D2: sham: $t_{(19)}=$ 3.049, $p<0.004$; lidocaine: $t_{(19)}=-0.582, p>0.284$, onetailed), whereas in the familiarity discrimination task, rats exhibited a significantly above chance bias for the novel object after either sham or lidocaine infusions (D1: sham: $t_{(19)}=3.696, p<$ 0.002; lidocaine: $t_{(19)}=2.908, p<0.005$; D2: sham: $t_{(19)}=3.810$, $p<0.001$; lidocaine: $t_{(19)}=3.346, p<0.002$, one-tailed). Finally, additional evidence indicating a deficit selective to the recency discrimination task comes from analyses showing that performance after lidocaine infusions was worse in the recency discrimination task than in the familiarity discrimination task in terms of both D1 $\left(t_{(19)}=2.713, p<0.02\right.$, two-tailed $)$ and D2 $\left(t_{(19)}=\right.$ 2.900, $p<0.01$, two-tailed) measures. Collectively, these data indicate that lidocaine infusions into the mPFC before the test trial disrupted the ability to discriminate the relative recency of previously explored objects but did not disrupt the ability to discriminate the relative familiarity of a previously explored from a novel object.

Infusions of lidocaine into the anterior Prh before test trials disrupted performance in both the familiarity and recency discrimination tasks. This was evidenced by a significant effect of infusion in terms of both D1 $\left(F_{(1,12)}=16.351, p=0.002\right)$ and D2 $\left(F_{(1,12)}=20.831, p=0.001\right)$ measures in the absence of a significant task by infusion interaction $\left(\mathrm{D} 1: F_{(1,12)}=0.315, p=0.585\right.$; D2: $\left.F_{(1,12)}=0.325, p=0.579\right)$. Subsequent planned comparisons further indicated that Prh infusions disrupted performance in both tasks as shown by a significant infusion effect in both the familiarity $\left(\mathrm{D} 1: t_{(12)}=3.963, p<0.002 ; \mathrm{D} 2: t_{(12)}=4.256, p<\right.$ $0.001)$ and recency $\left(\mathrm{D} 1: t_{(12)}=2.963, p<0.01 ; \mathrm{D} 2: t_{(12)}=2.144\right.$, $p<0.03$ ) discrimination tasks. Consistent with these findings, in both the familiarity and recency discrimination tasks, rats exhibited a significantly above chance bias for the novel/old familiar object after sham infusions (familiarity discrimination task: D1: $t_{(12)}=4.534, p<0.001$; D2: $t_{(12)}=6.506, p<0.001$; recency discrimination task: D1: $t_{(12)}=2.657, p<0.012$; D2: $t_{(12)}=$ 2.389, $p<0.018$ ) but not lidocaine infusions (familiarity discrimination task: D1: $t_{(12)}=0.328, p>0.374 ; \mathrm{D} 2: t_{(12)}=-0.199$, 
Table 2. Exploration times (in seconds; mean \pm SEM) by rats after sham, unilateral mPFC, unilateral Prh, or crossed unilateral mPFC plus Prh lidocaine infusions in experiment 2 (recency discrimination task) across trials

\begin{tabular}{lllll}
\hline Infusion & Trial 1 total & Trial 2 total & Trial 3 total & Trial 3 object $\mathrm{F}_{\mathrm{R}}$ \\
\hline Sham & $30.0 \pm 2.7$ & $35.3 \pm 4.4$ & $27.1 \pm 1.8$ & $16.6 \pm 0.9$ \\
mPFC & $40.9 \pm 3.8$ & $38.7 \pm 8.9$ & $25.3 \pm 2.4$ & $10.4 \pm 1.5$ \\
Prh & $36.5 \pm 3.9$ & $35.3 \pm 3.7$ & $26.8 \pm 2.9$ & $1.9 \pm 1.4$ \\
mPFC plus Prh & $35.4 \pm 2.5$ & $34.4 \pm 4.1$ & $25.1 \pm 3.7$ & 1.5 \\
\hline
\end{tabular}

$\mathrm{F}_{0}$, Older familiar object; $\mathrm{F}_{\mathrm{R}}$, more recent familiar object.

$p>0.422$; recency discrimination task: D1: $t_{(12)}=-0.899, p>$ $\left.0.192 ; \mathrm{D} 2: t_{(12)}=-0.129, p>0.498\right)$. Finally, additional evidence indicating a similar deficit in both tasks comes from analyses showing that performance after lidocaine infusions was not significantly different between tasks in terms of either D1 $\left(t_{(12)}=\right.$ $1.115 ; p>0.286$, two-tailed) or D2 $\left(t_{(12)}=-0.024 ; p>0.981\right.$, two-tailed) measures. Collectively, these data indicate that lidocaine infusions into the anterior Prh before test trials disrupted the ability to discriminate both the relative familiarity of a previously explored and novel object and the relative recency of two previously explored objects.

\section{Experiment 2}

Histology

All rats included in behavioral analyses had placements in the mPFC and anterior PRH similar to those included in experiment 1.

\section{Behavioral testing}

Raw exploration times across trials after sham, mPFC, Prh, and crossed mPFC plus Prh infusions are presented in Table 2. In general, most rats spent between 20 and $45 \mathrm{sec}$ in active exploration of the objects on all trials. The amount of exploration was not constant across trials but did not differ across infusion conditions as shown by a significant trial effect $\left(F_{(2,60)}=7.615 ; p=0.003\right)$ in the absence of a significant infusion effect $\left(F_{(3,60)}=0.665 ; p=\right.$ $0.580)$ or trial by infusion interaction $\left(F_{(6,60)}=0.676 ; p=0.669\right)$. Subsequent analyses (with data collapsed across infusion conditions) indicated that rats spent less total time exploring the objects in trial 3 than on trial $1\left(t_{(43)}=5.15 ; p<0.001\right)$ or trial 2 $\left.t_{(43)}=3.482 ; p<0.001\right)$ but did not differ in terms of exploration in trials 1 and $2\left(t_{(43)}=0.095 ; p=0.925\right)$. The reduced exploration time on trial 3 is not surprising, given that both objects are familiar on that trial whereas both objects are novel on trials 1 and 2. Overall, the above data suggest that infusions did not produce a gross impairment in exploratory behavior.

On test trials, data indicated that unilateral lidocaine infusions into the Prh produced a mild impairment of recency discrimination performance, whereas a significantly greater impairment was produced by crossed unilateral infusions into the $\mathrm{MPFC}$ and Prh (Table 2; Fig. 5A,B). Overall, the disruptive effect of lidocaine infusions was first indicated by a significant infusion effect in terms of both D1 $\left(F_{(3,30)}=4.213 ; p=0.013\right)$ and D2 $\left(F_{(3,30)}=\right.$ 5.420; $p=0.004)$ measures. Subsequent planned comparisons between infusion conditions then indicated the following: (1) unilateral mPFC infusions spared performance as shown by the absence of a significant difference between $\mathrm{mPFC}$ and sham infusions in terms of either D1 $\left(t_{(10)}=0.653 ; p>0.263\right.$, one-tailed $)$ and D2 $\left(t_{(10)}=0.518 ; p>0.307\right.$, one-tailed) measures; (2) unilateral Prh infusions produced a mild impairment as shown by a significant difference between Prh and sham infusions in terms of D2 measures $\left(t_{(10)}=1.943 ; p<0.041\right.$, one-tailed) but not D1 measures $\left(t_{(10)}=1.289 ; p>0.107\right.$, one-tailed); and (3) crossed unilateral infusions into the $\mathrm{mPFC}$ and Prh produced a more severe impairment as shown by significant differences between mPFC plus
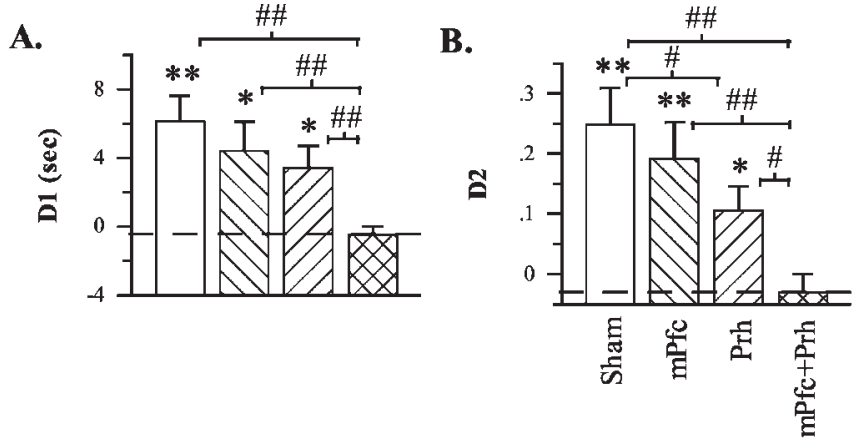

Figure 5. Differences in the amount of exploration directed at old familiar versus recent familiar (recency discrimination task) objects after sham, unilateral, or crossed unilateral infusions into anterior Prh and/or mPFC in experiment 2. A, Difference score (D1) calculated as time spent exploring the old familiar object less time spent exploring the recent familiar object. $B$, Weighted difference score (D2) calculated as D1 divided by the time spent exploring both objects. ${ }^{*} p<0.05$ and ${ }^{* *} p<0.01$, one-tailed, relative to chance performance (i.e., 0 , indicated by the dashed line); \#p $<0.05$ and \#\#p $<0.01$, one-tailed, sham versus lidocaine.

Prh infusions and all other treatment conditions in terms of both D1 measures (vs sham: $t_{(10)}=4.993, p<0.001$; vs mPFC: $t_{(10)}=2.760$, $p<0.010$; vs Prh: $t_{(10)}=3.143, p<0.005$; all tests one-tailed) and D2 measures (vs sham: $t_{(10)}=4.792, p<0.001$; vs mPFC: $t_{(10)}=3.228$, $p<0.005$; vs Prh: $t_{(10)}=2.974, p<0.008$, all tests one-tailed). Consistent with these findings, rats exhibited a significantly above chance bias for the old familiar object after sham (D1: $t_{(10)}=4.110$, $p<0.002$; D2: $t_{(10)}=4.164, p<0.002$; both tests one-tailed), unilateral mPFC (D1: $t_{(10)}=2.630, p<0.013$; D2: $t_{(10)}=3.067, p<$ 0.006 ; both tests one-tailed), and unilateral Prh (D1: $t_{(10)}=2.593$, $p<0.014$; D2: $\left.t_{(10)}=2.752, p<0.010\right)$ infusions but not after crossed mPFC plus Prh infusions (D1: $t_{(10)}=-0.884, p>0.198$; D2: $\left.t_{(10)}=-0.994, p>0.172\right)$. Collectively, these data indicate that crossed unilateral lidocaine infusions into the mPFC plus Prh before the test trial severely disrupted the ability to discriminate the relative recency of previously explored objects.

\section{Discussion}

Bilateral lidocaine infusions into the anterior Prh immediately before memory testing disrupted performance in two tests of spontaneous object exploration that required either familiarity or recency discrimination, whereas infusions into the $\mathrm{mPFC}$ disrupted recency, but not familiarity, discrimination. In a second experiment, crossed unilateral infusions into the mPFC and Prh disrupted recency discrimination, whereas unilateral infusions into either structure alone produced little or no effect.

\section{Prh contributions to object memory}

The present results provide additional support for the view that the Prh plays a critical role in discriminating the familiarity of objects (Aggleton and Brown, 1999; Murray and Richmond, 2001). Rats receiving lidocaine infusions into the Prh failed to 
direct more exploration at novel objects than familiar objects, yet did not exhibit any grossly observable behavioral abnormalities or altered overall amounts of object exploration. This pattern of behavior suggests that Prh inactivation specifically blocked capacity for familiarity discrimination, which agrees with other demonstrations of impairments after permanent Prh lesions in similar spontaneous object recognition tasks (Ennaceur et al., 1996; Aggleton et al., 1997; Ennaceur and Aggleton, 1997; Bussey et al., 1999; Liu and Bilkey, 2001; Mumby et al., 2002) and in reinforced tests of object recognition memory (Mumby and Pinel, 1994; Kesner et al., 2001; Murray and Richmond, 2001). The basis for impaired familiarity discrimination in the present study cannot be determined but could relate to the hypothesized role of the Prh in either object recognition memory per se (i.e., the ability to remember that an object has been previously encountered), object identification (i.e., the ability to perceive diverse object features as an integrated whole distinct from other similar objects) or both (Murray and Richmond, 2001). Regardless of the underlying basis for the impairment, our findings demonstrate that the Prh must be important during retrieval because inactivations were only produced before memory testing (i.e., during retrieval), leaving the Prh intact during encoding and maintenance (i.e., during and after the training trial). This Prh contribution to retrieval is further supported by previous findings that permanent Prh lesions produce retrograde amnesia for previously acquired object information (Myhrer and Wangen, 1996; Wiig et al., 1996; Thornton et al., 1997; Mumby and Glen, 2000), including information about object familiarity in the spontaneous object recognition task (Mumby et al., 2002).

The present results are the first to implicate the Prh cortex directly in recency discrimination performance. Rats receiving lidocaine infusions into the anterior Prh failed to direct more exploration at older familiar objects than more recent familiar objects, yet did not exhibit any grossly observable behavioral abnormalities or altered overall amounts of object exploration. This pattern of behavior suggests that Prh inactivation specifically blocked capacity for recency discrimination. This impairment could result from a disruption in temporal order memory per se or, because familiarity discrimination was also impaired, a disruption of object recognition memory or object identification. Regardless, as in the case with the familiarity discrimination task, our use of lidocaine inactivations just before memory testing demonstrates that the contribution of the Prh is required at the retrieval stage of temporal order memory processing. A final noteworthy point is that only the anterior Prh was directly inactivated in our study, which suggests that either this portion of the Prh is specifically important for performance of familiarity and recency discriminations or, more likely, that both types of discrimination, as assessed in the present study, are exquisitely sensitive to disruptions of Prh function.

\section{mPFC contributions to object memory}

In contrast to Prh inactivation, $\mathrm{mPFC}$ inactivation produced a selective pattern of effects across the two tasks. It disrupted the capacity to discriminate the relative recency of two familiar objects but failed to disrupt the capacity to discriminate the relative familiarity of a familiar object and a novel object. This selective impairment strongly suggests that MPFC inactivation produced a deficit specific to temporal order memory. Performance on both tasks was assessed using identical test trial format and behavioral indices of performance; therefore, sparing of familiarity discrimination task performance indicates that neither a disruption of sensory/perceptual, motivational, motor/exploratory, or atten- tional components of performance nor a failure to recognize or discriminate objects was the basis for the recency discrimination task deficit. This indication of a specific role for the mPFC in object temporal order memory is consistent with findings that permanent mPFC lesions also produce a selective temporal order memory deficit in similar spontaneous object exploration tasks (Mitchell and Laiacona, 1998). A general role for the PFC in temporal order memory is further indicated by findings that PFC damage disrupts performance on spatial temporal order memory tasks in rats (Kesner and Holbrook, 1987; Chiba et al., 1994, 1997; Hannesson et al., 2004), on object temporal order memory tasks in monkeys (Petrides, 1991), and on temporal order memory tasks using words, spatial locations, or pictures in humans (Lewinsohn et al., 1972; Shimamura et al., 1990; McAndrews and Milner, 1991; Kopelman et al., 1997). An additional implication of our results is that, given the use of lidocaine inactivations just before memory testing, the PFC must contribute to processes related to object temporal order memory retrieval.

The role of the PFC in object recognition is not as clear. The present findings suggest that the PFC does not play a role in the retrieval or use of information necessary for discriminating object familiarity, at least when used to guide spontaneous exploratory behavior. This sparing is unlikely to result from inefficacy of our brain manipulation, because the same manipulation effectively disrupted recency discrimination performance, or insensitivity of our recognition task, because this same task was sensitive to Prh inactivation. Moreover, our results are supported by findings that permanent mPFC lesions also spared performance in the spontaneous object recognition task (Mitchell and Laiacona, 1998; Yee, 2000) and that PFC damage in humans produced a deficit in temporal order memory accompanied by relative sparing of recognition memory (Lewinsohn et al., 1972; Shimamura et al., 1990; McAndrews and Milner, 1991; Kesner et al., 1994; Shaw and Aggleton, 1995; Kopelman et al., 1997).

Other results, however, suggest that the PFC does play a role in object recognition memory. For example, object recognition deficits have been observed in object delayed nonmatch-to-sample tasks after damage to the orbital and/or anterior cingulate cortex in primates (Bachevalier and Mishkin, 1986; Meunier et al., 1997) and in either a delayed nonmatch-to-sample task or a continuous object matching task after damage to the medial or ventromedial PFC in rats (Kolb et al., 1994; Kesner et al., 1996; Ragozzino et al., 2002). Indeed, it has been suggested that circuitry linking the Prh, mediodorsal thalamus, and ventrolateral PFC in the primate is critical for object recognition memory (Meunier et al., 1997; Parker and Gaffan, 1998).

There are a number of factors that may account for the differences between the deficits in object recognition seen in reinforced paradigms and the sparing of object recognition seen in spontaneous paradigms. First, in contrast to spontaneous tasks, reinforced object recognition tasks require: (1) extensive training (and thus the retention and application of considerable procedural information); (2) appetitive reinforcement; (3) rapid encoding (because the object is only viewed for seconds); and (4) short-term memory, which is assessed across delays of seconds to a few minutes. There is evidence suggesting that the PFC plays a particular role in functions related to all of these factors (Goldman-Rakic, 1996; Kesner, 2000; Cardinal et al., 2002), and thus it is possible that the involvement of the PFC in reinforced object recognition paradigms relates critically to its role in some, or all, of these components. In this regard, comparisons of the effects of PFC lesions across spontaneous and reinforced object recognition tasks in which encoding and retention durations 
were more closely equated may prove informative. An additional factor that may be critical is the location and extent of damage within the PFC. The primate studies that have found deficits highlight the potential importance of the orbital region (Meunier et al., 1997), whereas the rat studies indicate that substantial damage to both the prelimbic and infralimbic cortex (i.e., the ventromedial PFC) may be critical (Ragozzino et al., 2002). Thus, it is worth noting that maintained capacity for the spontaneous object recognition task, including in the present study, has been observed after lesions that have spared some, or all, of the infralimbic cortex and spared orbital regions of the PFC almost entirely (Mitchell and Laiacona, 1998; Yee, 2000).

\section{Prh-mPFC interactions in object memory}

The results of experiment 2 demonstrate that the Prh and mPFC play an interactive role in object temporal order memory. Unilateral inactivation of either structure alone produced little or no disruption of recency discrimination, demonstrating that unilateral activity within each structure is sufficient to support performance. However, crossed unilateral inactivations of the anterior Prh and PFC greatly impaired performance. Because unilateral interactions between the anterior Prh (or mPFC) and all other structures, except the mPFC (or Prh), remained intact with this procedure, these findings suggest that intrahemispheric interactions specifically between the Prh and mPFC must be critical for performance. These are the first results, then, to indicate an important role for Prh-mPFC interactions in memory in the rat and are consistent with findings in primates that also suggest that the Prh and PFC must interact intrahemispherically for some, but not all, types of object memory (Parker and Gaffan, 1998). Interestingly, these results also parallel our previous findings that the hippocampus and $\mathrm{MPFC}$ must interact within the same hemisphere to support higher-order forms of spatial memory (Floresco et al., 1997, 1999).

The exact route by which Prh-mPFC interactions occur cannot be determined from the present results. There are direct reciprocal connections between the Prh and mPFC that are greater ipsilaterally and from anterior than posterior Prh (Sesack et al., 1989; McIntyre et al., 1996; Burwell, 2000; Delatour and Witter, 2002). However, studies in primates suggest that direct connections between these and closely related regions are not essential to support interaction-dependent object memory functions (Parker and Gaffan, 1998; Easton et al., 2001). Alternatively, there are several bisynaptic routes of interaction, for example, involving the mediodorsal thalamus and basal forebrain (Burwell, 2000), and some evidence indeed suggests that these routes (e.g., basal forebrain) may be particularly important (Easton et al., 2001). Additional studies, however, are needed to specify the critical circuits and routes of interaction that support temporal order as well as other forms of memory for objects.

\section{Conclusion}

Based on the pattern of results in the present study, the roles of the Prh and mPFC in memory for objects can be hypothesized as follows. The Prh contributes to retrieval of information about previously encountered objects that is essential when assessing familiarity. This information then is also used when additional mnemonic processing of familiar objects is required (e.g., in temporal order memory), which involves interactions between the Prh and PFC within the same hemisphere. This hypothesis is consistent with broader theories of memory circuits proposing that attribute information is stored in posterior forebrain sites, such as the Prh and hippocampus, and is accessed and further processed via interactions with the PFC to enable working memory-dependant higher-order cognitive and mnemonic functions (Kesner, 1998; Mayes and Roberts, 2001). Further study of the roles of the Prh, PFC, and related structures in object memory using spontaneous and reinforced tasks can provide important additional insights into the organization of memory processing in the brain and may contribute to our understanding of the pathophysiology of memory disorders in which these circuits are compromised.

\section{References}

Aggleton JP, Brown MW (1999) Episodic memory, amnesia, and the hippocampal-anterior thalamic axis. Behav Brain Sci 22:425-444.

Aggleton JP, Keen S, Warburton EC, Bussey TJ (1997) Extensive cytotoxic lesions involving both the rhinal cortices and area TE impair recognition but spare spatial alternation in the rat. Brain Res Bull 43:279-287.

Bachevalier J, Mishkin M (1986) Visual recognition impairment follows ventromedial but not dorsolateral prefrontal lesions in monkeys. Behav Brain Res 20:249-261.

Brown MW (1996) Neuronal responses and recognition memory. Semin Neurosci 8:23-32.

Brown MW, Bashir ZI (2002) Evidence concerning how neurons of the perirhinal cortex may effect familiarity discrimination. Philos Trans R Soc Lond B Biol Sci 357:1083-1095.

Burwell RD (2000) The parahippocampal region: corticocortical connectivity. Ann NY Acad Sci 911:25-42.

Burwell RD (2001) Borders and cytoarchitecture of the perirhinal and postrhinal cortices in the rat. J Comp Neurol 437:17-41.

Bussey TJ, Muir JL, Aggleton JP (1999) Functionally dissociating aspects of event memory: the effects of combined perirhinal and postrhinal cortex lesions on object and place memory in the rat. J Neurosci 19:495-502.

Cardinal RN, Parkinson JA, Hall J, Everitt BJ (2002) Emotion and motivation: the role of the amygdala, ventral striatum, and prefrontal cortex. Neurosci Biobehav Rev 26:321-352.

Chiba AA, Kesner RP, Reynolds AM (1994) Memory for spatial location as a function of temporal lag in rats: role of hippocampus and medial prefrontal cortex. Behav Neural Biol 61:123-131.

Chiba AA, Kesner RP, Gibson CJ (1997) Memory for temporal order of new and familiar spatial location sequences: role of the medial prefrontal cortex. Learn Mem 4:311-317.

Delatour B, Witter MP (2002) Projections from the parahippocampal region to the prefrontal cortex in the rat: evidence of multiple pathways. Eur J Neurosci 15:1400-1407.

Easton A, Parker A, Gaffan D (2001) Crossed unilateral lesions of medial forebrain bundle and either inferior temporal or frontal cortex impair object recognition memory in rhesus monkeys. Behav Brain Res 121:1-10.

Ennaceur A, Aggleton JP (1997) The effects of neurotoxic lesions of the perirhinal cortex combined to fornix transection on object recognition memory in the rat. Behav Brain Res 88:181-193.

Ennaceur A, Delacour J (1988) A new one-trial test for neurobiological studies of memory in rats. I: Behavioral data. Behav Brain Res $31: 47-59$

Ennaceur A, Neave N, Aggleton JP (1996) Neurotoxic lesions of the perirhinal cortex do not mimic the behavioural effects of fornix transection in the rat. Behav Brain Res 80:9-25.

Floresco SB, Seamans JK, Phillips AG (1997) Selective roles for hippocampal, prefrontal cortical, and ventral striatal circuits in radial-arm maze tasks with or without a delay. J Neurosci 17:1880-1890.

Floresco SB, Braaksma DN, Phillips AG (1999) Thalamic-cortical-striatal circuitry subserves working memory during delayed responding on a radial arm maze. J Neurosci 19:11061-11071.

Funahashi S, Inoue M, Kubota K (1997) Delay-period activity in the primate prefrontal cortex encoding multiple spatial positions and their order of presentation. Behav Brain Res 84:203-223.

Goldman-Rakic PS (1996) The prefrontal landscape: implications of functional architecture for understanding human mentation and the central executive. Philos Trans R Soc Lond B Biol Sci 351:1445-1453.

Hannesson DK, Vacca G, Howland JG, Phillips AG (2004) Medial prefrontal cortex is involved in spatial temporal order memory but not spatial 
recognition memory in tests relying on spontaneous exploration in the rat. Behav Brain Res, in press.

Kesner RP (1998) Neural mediation of memory for time: role of the hippocampus and medial prefrontal cortex. Psychonomic Bull Rev 5:585-596.

Kesner RP (2000) Subregional analysis of mnemonic functions of the prefrontal cortex in the rat. Psychobiology 28:219-228.

Kesner RP, Holbrook T (1987) Dissociation of item and order spatial memory in rats following medial prefrontal cortex lesions. Neuropsychologia 25:653-664.

Kesner RP, Hopkins RO, Fineman B (1994) Item and order dissociation in humans with prefrontal cortex damage. Neuropsychologia 32:881-891.

Kesner RP, Hunt ME, Williams JM, Long JM (1996) Prefrontal cortex and working memory for spatial response, spatial location, and visual object information in the rat. Cereb Cortex 6:311-318.

Kesner RP, Ravindranathan A, Jackson P, Giles R, Chiba AA (2001) A neural circuit analysis of visual recognition memory: role of perirhinal, medial, and lateral entorhinal cortex. Learn Mem 8:87-95.

Kesner RP, Gilbert PE, Barua LA (2002) The role of the hippocampus in memory for the temporal order of a sequence of odors. Behav Neurosci 116:286-290.

Kolb B, Buhrmann K, McDonald R, Sutherland RJ (1994) Dissociation of the medial prefrontal, posterior parietal, and posterior temporal cortex for spatial navigation and recognition memory in the rat. Cereb Cortex $4: 664-680$.

Kopelman MD, Stanhope N, Kingsley D (1997) Temporal and spatial context memory in patients with focal frontal, temporal lobe, and diencephalic lesions. Neuropsychologia 35:1533-1545.

Lalonde R, Hannequin D (1999) The neurobiological basis of time estimation and temporal order. Rev Neurosci 10:151-173.

Lewinsohn PM, Zieler RE, Libet J, Eyeberg S, Nielson G (1972) Short-term memory: a comparison between frontal and nonfrontal right- and lefthemisphere brain-damaged patients. J Comp Physiol Psychol 81:248-255.

Liu P, Bilkey DK (2001) The effect of excitotoxic lesions centered on the hippocampus or perirhinal cortex in object recognition and spatial memory tasks. Behav Neurosci 115:94-111.

Mayes AR, Roberts N (2001) Theories of episodic memory. Philos Trans R Soc Lond B Biol Sci 356:1395-1408.

McAndrews MP, Milner B (1991) The frontal cortex and memory for temporal order. Neuropsychologia 29:849-859.

McIntyre DC, Kelly ME, Staines WA (1996) Efferent projections of the anterior perirhinal cortex in the rat. J Comp Neurol 369:302-318.

Meunier M, Bachevalier J, Mishkin M (1997) Effects of orbital frontal and anterior cingulate lesions on object and spatial memory in rhesus monkeys. Neuropsychologia 35:999-1015.

Mitchell JB, Laiacona J (1998) The medial frontal cortex and temporal memory: tests using spontaneous exploratory behaviour in the rat. Behav Brain Res 97:107-113.

Mumby DG, Glenn MJ (2000) Anterograde and retrograde memory for object discriminations and places in rats with perirhinal cortex lesions. Behav Brain Res 114:119-134.
Mumby DG, Pinel JP (1994) Rhinal cortex lesions and object recognition in rats. Behav Neurosci 108:11-18.

Mumby DG, Glenn MJ, Nesbitt C, Kyriazis DA (2002) Dissociation in retrograde memory for object discriminations and object recognition in rats with perirhinal cortex damage. Behav Brain Res 132:215-226.

Murray EA, Richmond BJ (2001) Role of perirhinal cortex in object perception, memory, and associations. Curr Opin Neurobiol 11:188-193.

Myhrer T, Wangen K (1996) Marked retrograde and anterograde amnesia of a visual discrimination task in rats with selective lesions of the perirhinal cortex. Neurobiol Learn Mem 65:244-252.

Ninokura Y, Mushiake H, Tanji J (2003) Representation of the temporal order of visual objects in the primate lateral prefrontal cortex. J Neurophysiol 89:2868-2873.

Parker A, Gaffan D (1998) Interaction of frontal and perirhinal cortices in visual object recognition memory in monkeys. Eur J Neurosci 10:3044-3057.

Paxinos G, Watson C (1998) The rat brain in stereotaxic coordinates. Toronto: Academic.

Petrides M (1991) Functional specialization within the dorsolateral frontal cortex for serial order memory. Proc R Soc Lond B Biol Sci 246:299-306.

Ragozzino ME, Detrick S, Kesner RP (2002) The effects of prelimbic and infralimbic lesions on working memory for visual objects in rats. Neurobiol Learn Mem 77:29-43.

Rainer G, Miller EK (2000) Effects of visual experience on the representation of objects in the prefrontal cortex. Neuron 27:179-189.

Seamans JK, Floresco SB, Phillips AG (1995) Functional differences between the prelimbic and anterior cingulate regions of the rat prefrontal cortex. Behav Neurosci 109:1063-1073.

Sesack SR, Deutch AY, Roth RH, Bunney BS (1989) Topographical organization of the efferent projections of the medial prefrontal cortex in the rat: an anterograde tract-tracing study with Phaseolus vulgaris leucoagglutinin. J Comp Neurol 290:213-242.

Shaw C, Aggleton JP (1995) Evidence for the independence of recognition and recency memory in amnesic subjects. Cortex 31:57-71.

Shimamura AP, Janowsky JS, Squire LR (1990) Memory for the temporal order of events in patients with frontal lobe lesions and amnesic patients. Neuropsychologia 28:803-813.

Tehovnik EJ, Sommer MA (1997) Effective spread and timecourse of neural inactivation caused by lidocaine injection in monkey cerebral cortex. J Neurosci Methods 74:17-26.

Thornton JA, Rothblat LA, Murray EA (1997) Rhinal cortex removal produces amnesia for preoperatively learned discrimination problems but fails to disrupt postoperative acquisition and retention in rhesus monkeys. J Neurosci 17:8536-8549.

Wiig KA, Cooper LN, Bear MF (1996) Temporally graded retrograde amnesia following separate and combined lesions of the perirhinal cortex and fornix in the rat. Learn Mem 3:313-325.

Yee BK (2000) Cytotoxic lesion of the medial prefrontal cortex abolishes the partial reinforcement extinction effect, attenuates prepulse inhibition of the acoustic startle reflex and induces transient hyperlocomotion, while sparing spontaneous object recognition memory in the rat. Neuroscience 95:675-689. 